\title{
Quantification of Photocyanine in Human Serum by High-Performance Liquid Chromatography-Tandem Mass Spectrometry and Its Application in a Pharmacokinetic Study
}

\author{
Bing-Tian Bi, ${ }^{1,2}$ Ben-Yan Zou, ${ }^{1,3}$ Li-Ting Deng, ${ }^{1,2}$ Jing Zhan,,2 \\ Hai Liao, ${ }^{1,2}$ Kun-Yao Feng, ${ }^{1,2}$ and $\mathrm{Su} \mathrm{Li}^{1,2}$ \\ ${ }^{1}$ State Key Laboratory of Oncology in South China, Collaborative Innovation Center of Cancer Medicine, \\ Sun Yat-sen University Cancer Center, Guangzhou, Guangdong 510060, China \\ ${ }^{2}$ Department of Clinical Trial Center, Cancer Center, Sun Yat-sen University, Guangzhou, Guangdong 510060, China \\ ${ }^{3}$ Department of Medical Oncology, Cancer Center, Sun Yat-sen University, Guangzhou, Guangdong 510060, China
}

Correspondence should be addressed to Su Li; lisu@sysucc.org.cn

Received 14 February 2014; Accepted 3 June 2014; Published 24 June 2014

Academic Editor: Xiu-Ping Yan

Copyright (C) 2014 Bing-Tian Bi et al. This is an open access article distributed under the Creative Commons Attribution License, which permits unrestricted use, distribution, and reproduction in any medium, provided the original work is properly cited.

\begin{abstract}
Photocyanine is a novel anticancer drug. Its pharmacokinetic study in cancer patients is therefore very important for choosing doses, and dosing intervals in clinical application. A rapid, selective and sensitive high-performance liquid chromatographytandem mass spectrometry (HPLC-MS/MS) method was developed and validated for the determination of photocyanine in patient serum. Sample preparation involved one-step protein precipitation by adding methanol and N,N-dimethyl formamide to $0.1 \mathrm{~mL}$ serum. The detection was performed on a triple quadrupole tandem mass spectrometer operating in multiple reaction-monitoring (MRM) mode. Each sample was chromatographed within $7 \mathrm{~min}$. Linear calibration curves were obtained for photocyanine at a concentration range of $20-2000 \mathrm{ng} / \mathrm{mL}(r>0.995)$, with the lower limit of quantification (LLOQ) being $20 \mathrm{ng} / \mathrm{mL}$. The intrabatch accuracy ranged from $101.98 \%$ to $107.54 \%$, and the interbatch accuracy varied from $100.52 \%$ to $105.62 \%$. Stability tests showed that photocyanine was stable throughout the analytical procedure. This study is the first to utilize the HPLC-MS/MS method for the pharmacokinetic study of photocyanine in six cancer patients who had received a single dose of photocyanine $(0.1 \mathrm{mg} / \mathrm{kg})$ administered intravenously.
\end{abstract}

\section{Introduction}

Photodynamic therapy (PDT) is a potential model for cancer therapy, which has been used to treat or relieve the symptoms of skin cancer, esophageal cancer, prostate cancer, and nonsmall cell lung cancer [1-3]. During the PDT procedure, the excited photosensitizer forms highly reactive oxygen species using visible light of an appropriate wavelength, resulting in oxidative damage to cellular membranes and membranous organelles $[2,4-7]$.

As one type of the photosensitizer, porphyrins have been approved for PDT in the USA, Europe, Canada, and Japan, but their weak absorption attenuates their optimal application in PDT [8]. Phthalocyanines and their derivatives are also widely used photosensitizers for the PDT of cancer, displaying high absorption of visible light, mainly in the phototherapeutic wavelength window $(600-800 \mathrm{~nm})[9,10]$. Photosense, Pc4, and CGP55847 are second-generation photosensitizers that have been used in clinical practice [11-14].

Photocyanine (ZnPcS2P2), a new second-generation PDT drug, was approved for clinical trials as a new medicine in 2008 by the State Food and Drug Administration in China. It is an isomeric mixture of di-(potassium sulfonate)-diphthalimidomethyl phthalocyanine zinc (Figure 1) [15-17]. The presence of both hydrophobic and hydrophilic groups in photocyanine would improve its tumor selectivity. Clinical trial of a novel synthesized drug requires a reliable method for measuring levels of the drug in biological samples. Because photocyanine is a novel photodynamic drug, few methods have been developed for its quantification. Li et al. [18] also 


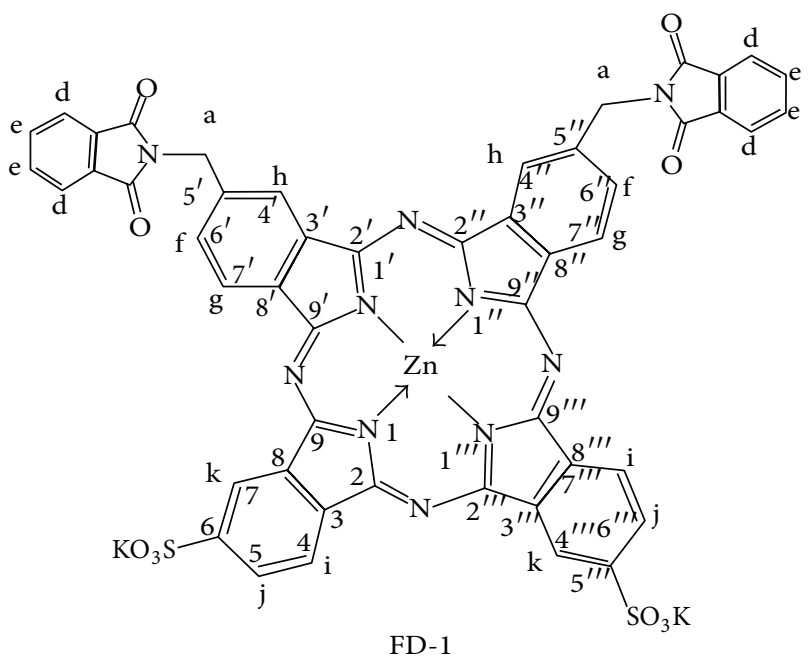

FD-1

(a)

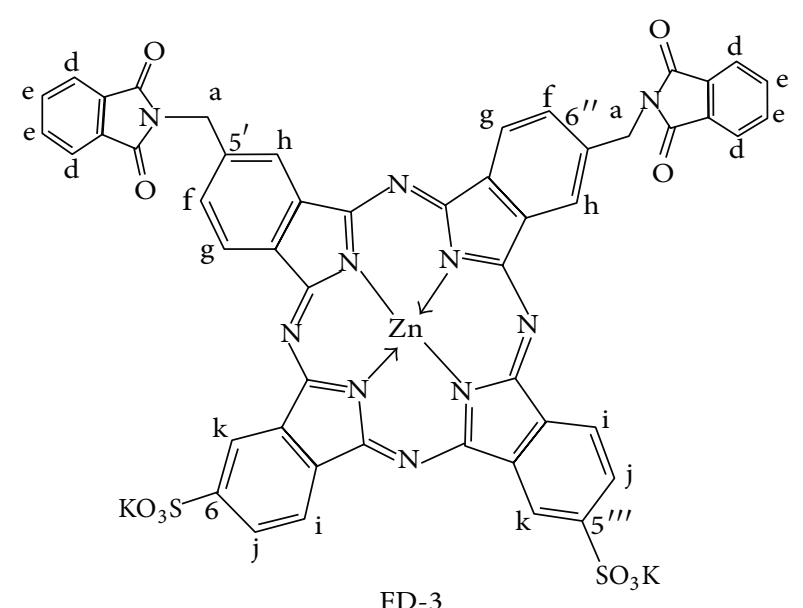

(c)

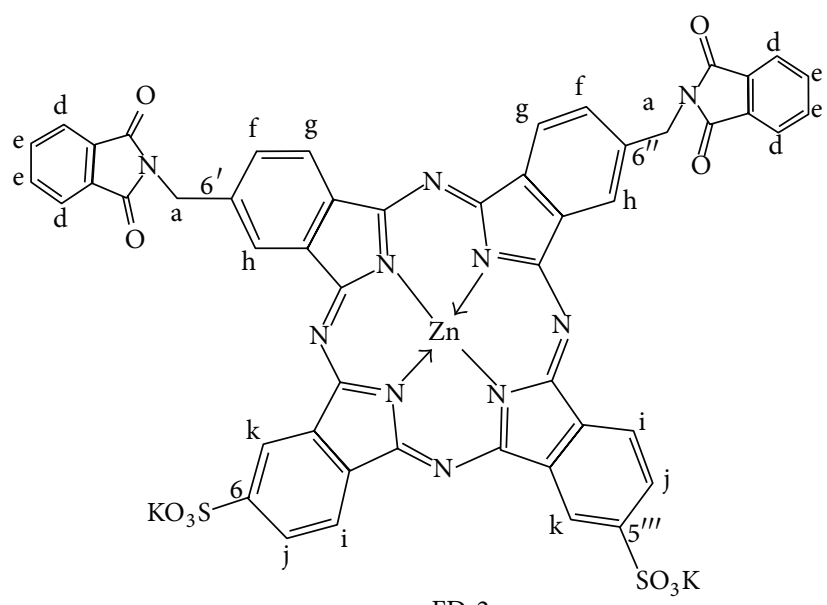

FD-2

(b)

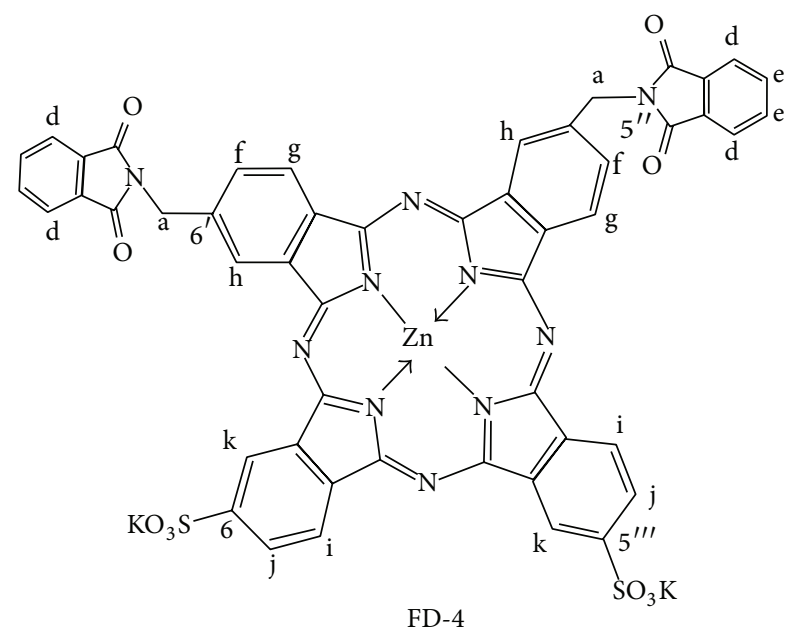

(d)

Figure 1: Chemical structures of photocyanine.

reported an HPLC method to separate the four isomers of a photocyanine mixture from human serum. However, the detection signals from this method display insufficient specificity in the biological samples, because it is difficult to avoid the interference from the matrix or other interferents by ultraviolet detector. Therefore, a method with higher specificity should be established to ensure the validity of the determination.

No evidence verifies the difference of each isomer of photocyanine in pharmacodynamic studies. Moreover, no standard substances for any of the isomers can be obtained from Fujian Longhua Pharmaceutical Co. Therefore, we developed a new high-performance liquid chromatographytandem mass spectrometry (HPLC-MS/MS) method to determine the total concentration of photocyanine in cancer patient serum in a pharmacokinetic study. The method was validated for its specificity, sensitivity, linearity, accuracy, precision, matrix effect, dilution integrity, and stability, and the data established the method as a high-throughput and reliable bioanalytical assay.

\section{Materials and Methods}

2.1. Experimental Chemicals. Photocyanine (purity > 95\%) and the internal standard (IS) mono- $\beta$-sulfonated zinc phthalocyanine potassium (purity $>95 \%$ ) were provided by Fujian Longhua Pharmaceutical Co. (Fujian, China). HPLCgrade $\mathrm{N}, \mathrm{N}$-dimethyl formamide (DMF) and methanol were purchased from Tedia Company, Inc., (Fairfield, OH, USA). Aqueous ammonia was obtained from Guangzhou Chemical Reagent Factory (Guangzhou, Guangdong, China). Deionized water was obtained from a Milli-Q analytical deionization system (Millipore, Bedford, MA, USA). Freshly obtained, drug-free human serum was collected from healthy individuals and stored at $-80^{\circ} \mathrm{C}$ before use. 
2.2. Chromatographic Conditions. The HPLC system consisted of an LC-20AD solvent delivery system, an SIL-20AC autosampler, a CTO-20AC column oven, and a CBM-20A controller from Shimadzu (Kyoto, Japan). Chromatographic separation of photocyanine and mono- $\beta$-sulfonated zinc phthalocyanine potassium was evaluated on an XBridge C18 column $(50 \mathrm{~mm} \times 4.6 \mathrm{~mm}, 5 \mu \mathrm{m})$ from Waters (Milford, MA, USA). For method validation and sample analysis, chromatographic separation was conducted by gradient elution using deionized water (adjusted to $\mathrm{pH} 10.0$ with aqueous ammonia) as mobile phase A (MPA) and methanol as mobile phase $\mathrm{B}$ (MPB). The HPLC program for gradient elution was as follows: $20 \%$ of MPB ( $0-0.2 \mathrm{~min}$ ), from $20 \%$ to $95 \%$ of MPB $(0.2-1.3 \mathrm{~min}), 95 \%$ of MPB (1.3-4.0 min), from $95 \%$ to $20 \%$ of MPB (4.0-4.1 min), and 20\% of MPB (4.1-7.0 min). The separation was performed using a flow rate of $0.6 \mathrm{~mL} / \mathrm{min}$. The column temperature was maintained at $60^{\circ} \mathrm{C}$.

\subsection{Mass Spectrometric Conditions. An API 4000 QTRAP} system (AB SCIEX, MA, USA) was operated in negative ionization mode with multiple reaction monitoring (MRM) for HPLC-MS/MS analysis. The mass spectrometric parameters were optimized to improve the MRM sensitivity. The instrument parameters for monitoring photocyanine and IS were as follows: vaporizer temperature, $650^{\circ} \mathrm{C}$; ion spray voltage, $-4,500 \mathrm{~V}$; curtain gas (CUR), nitrogen, 25 ; nebulizing gas (GS1), 65; heated gas (GS2), 65; declustering potential (DP), photocyanine $-140 \mathrm{~V}$, IS $-135 \mathrm{~V}$; collision energy (CE), photocyanine $-50 \mathrm{eV}$, IS $-64.4 \mathrm{eV}$; entrance potential (EP), $-10 \mathrm{~V}$; collision cell exit potential (CXP), $-10 \mathrm{~V}$. The precursor-to-product ion transitions used for the MRM of photocyanine and IS were $m / z 526.0 \rightarrow 146.0$ and $m / z 655.1$ $\rightarrow 591.8$, respectively. The mass spectrometer was operated at unit mass resolution for both the first and third quadrupoles.

2.4. Sample Preparation. A $100 \mu \mathrm{L}$ aliquot of blank human serum, spiked serum, or pharmacokinetic study serum was transferred to a $1.5 \mathrm{~mL}$ Eppendorf tube. Then, $200 \mu \mathrm{L}$ of DMF was added to each tube of serum, and the mixture was vortexed for $1 \mathrm{~min}$. The mixture was then spiked with $300 \mu \mathrm{L}$ methanol containing $450 \mathrm{ng} / \mathrm{mL}$ IS, vortexed, and centrifuged for $10 \mathrm{~min}$ at $15,000 \mathrm{rpm}$ at $4^{\circ} \mathrm{C}$. The supernatant was collected and filtered. $10 \mu \mathrm{L}$ of supernatant was injected into the LC-MS/MS system for analysis.

2.5. Method Validation. Photocyanine was validated for an HPLC-MS/MS assay. Specificity, the lower limits of quantification (LLOQ), linearity, accuracy, precision, extraction recovery, matrix effect, and stability were evaluated during method validation. The specificity was assessed by testing six independent aliquots of blank serum for exclusion of any endogenous interference at the peak region of photocyanine or IS (Figure 2). LLOQ was defined as the lowest concentration on the standard calibration curve from six different batches, in which both precision and accuracy were $\leq 20 \%$ with a signal-to-noise ratio $(\mathrm{S} / \mathrm{N})>10$. The linearity of the calibration curve was evaluated over the range of $20 \mathrm{ng} / \mathrm{mL}$ and $2000 \mathrm{ng} / \mathrm{mL}$. Calibration curves were constructed via linear least-squares regression analysis by plotting the peakarea ratios (photocyanine/IS) versus the drug concentrations in the serum, and the resulting correlation coefficient $(r>$ 0.99) was considered satisfactory. Precision and accuracy were assessed by the analytes covering the range of the calibration curve, in which the criteria for acceptability are defined as an accuracy $\pm 15 \%$ standard deviation (SD) from the nominal values and a precision of $\pm 15 \%$ relative standard deviation (RSD). Intrabatch accuracy and precision were evaluated by analyzing the quality control (QC) samples at concentrations of 60,1000 , and $1600 \mathrm{ng} / \mathrm{mL}$ with six duplicated levels per concentration on the same day. The interbatch accuracy and precision were assessed over three days. The extraction recovery of photocyanine and IS was determined by calculating the ratio of the peak area of photocyanine and IS spiked in serum before extraction against postextraction spiked photocyanine and IS at the same concentration. The matrix effect was determined by calculating the matrix factor, which was obtained as the ratio of the analyte peak response in the presence of matrix ions to the analyte peak response in the absence of matrix ions by spiking analytes into blank serum extracts and blank water extracts. The stability of photocyanine was compared to the nominal level of photocyanine to determine whether photocyanine was stable in the experiments, including postpreparative stability test, freeze-thaw cycle test, and long-term stability test. If the calculated concentration of photocyanine was less than the nominal concentration by $>15 \%$, the analyte was considered to be unstable. Low, medium, and high serum QC samples were determined in six duplicated levels. The stability of the extracts was evaluated by putting them at room temperature for $24 \mathrm{~h}$ and then subjecting them to the analytical procedure. Photocyanine maintained at $-80^{\circ} \mathrm{C}$ for 30 days was evaluated by comparing the postfreeze measured concentration with the initial concentration added to the sample. The freeze-thaw stability of the samples was assessed over three freeze-thaw cycles by thawing samples at room temperature, refreezing them for $24 \mathrm{~h}$ at $-80^{\circ} \mathrm{C}$ and then analyzing them.

2.6. Application. Six patients with cancer were enrolled at the Cancer Center, Sun Yat-sen University. The patients were five males and one female, ranging in age from 37 to 69 years, who had been diagnosed with a primary or metastatic malignancy. All patients provided written informed consent prior to participation. The patients were infused (i.v. administration) with photocyanine $(0.1 \mathrm{mg} / \mathrm{kg})$ for $60 \mathrm{~min}$. Blood samples were obtained at $0,0.5,1,2,4,6,8,12,24,72,120$, and $168 \mathrm{~h}$ after administration and then placed on ice and kept away from light. The blood samples were centrifuged at $3000 \mathrm{rpm} / \mathrm{min}$ for $10 \mathrm{~min}$, and the serum was stored at $-80^{\circ} \mathrm{C}$ until the analysis was conducted. The present study was approved by the Human Subjects Review Committees of the University of Sun Yat-sen University Cancer Center and conducted according to the Declaration of Helsinki.

Noncompartmental pharmacokinetic parameters were calculated using WinNonlin (Version 5.0, PUMCH Clinical Pharmacology Research Center). The maximum serum concentration $\left(C_{\max }\right)$ and time to reach it $\left(T_{\max }\right)$ were determined 


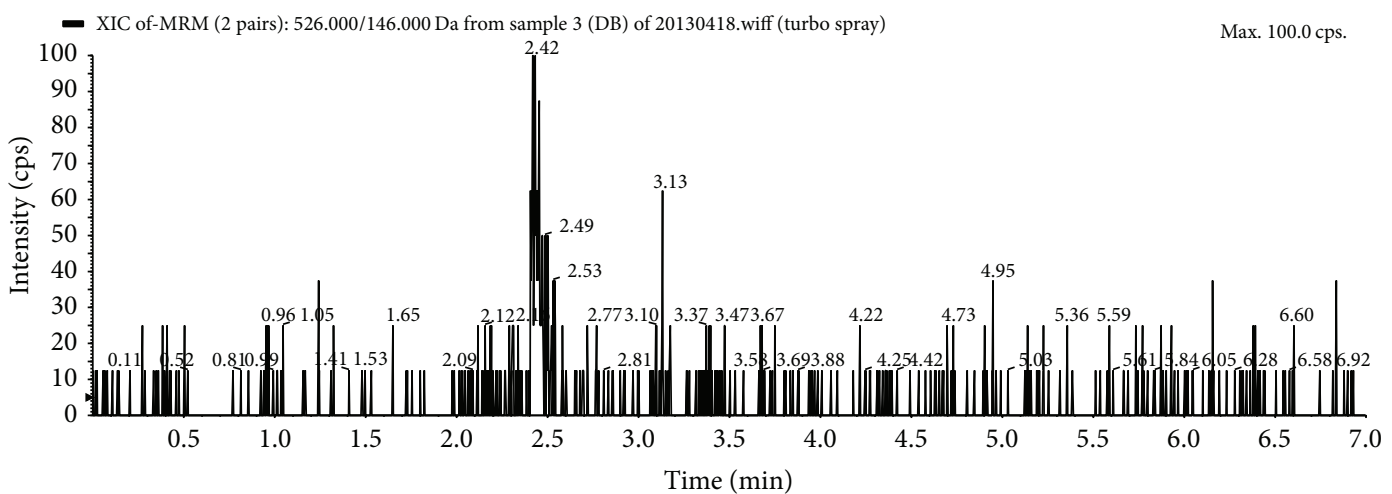

(a)

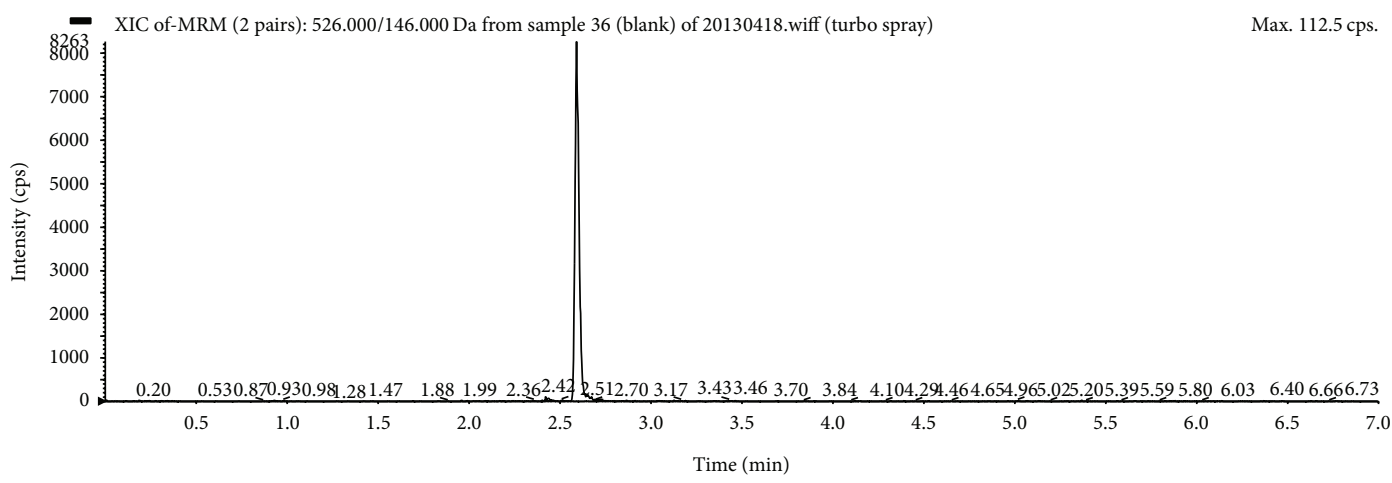

(b)

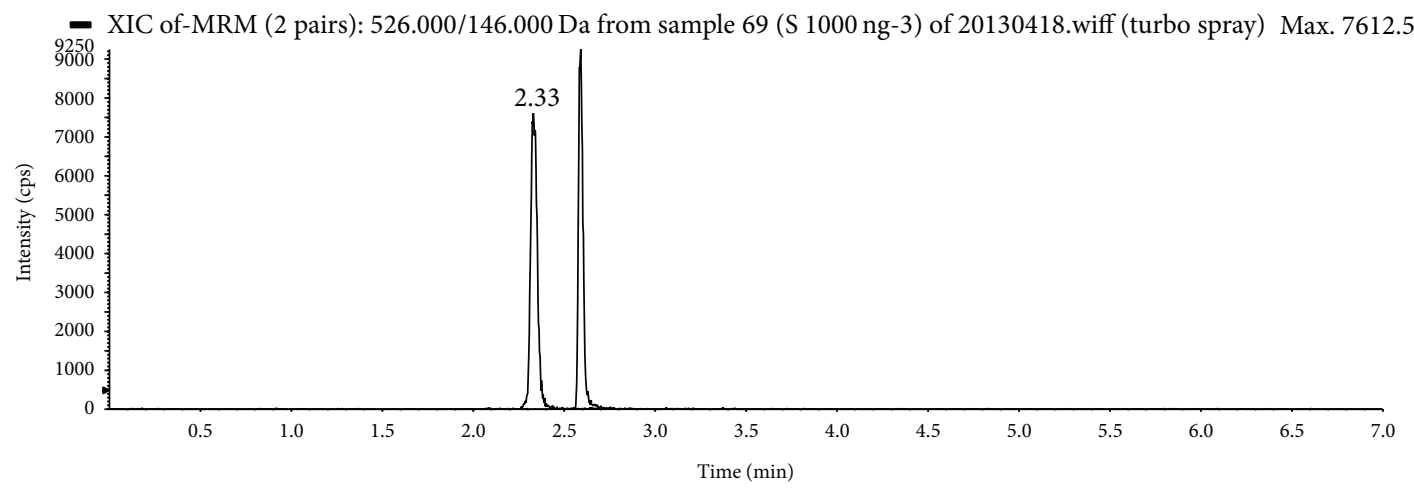

(c)

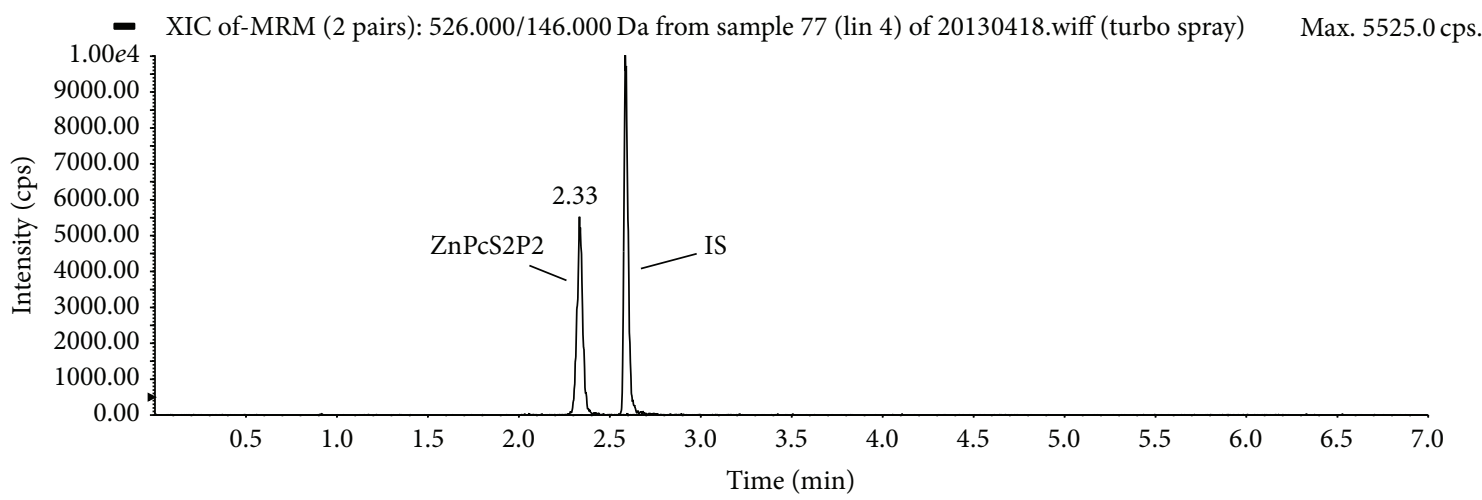

(d)

Figure 2: Representative HPLC-MS/MS chromatograms of extract from (a) blank serum; (b) a human serum sample spiked with mono- $\beta$ sulfonated zinc phthalocyanine potassium (IS), (c) a human serum sample spiked with $1000 \mathrm{ng} / \mathrm{mL}$ photocyanine and IS, and (d) a patient serum sample after i.v. administration of photocyanine spiked with IS. 
TABLE 1: Peak symmetry factor of photocyanine in different percentage of mobile phase $\mathrm{B}$.

\begin{tabular}{lcc}
\hline MPB (\%) & Symmetry factor $(n=5)$ & RSD (\%) \\
\hline 95 & 1.052 & 4.67 \\
90 & 1.103 & 9.00 \\
80 & 1.198 & 7.45 \\
70 & 1.354 & 7.67 \\
\hline
\end{tabular}

directly from the data. The terminal-phase rate constant $(\lambda z)$ was calculated as the negative slope of the log-linear terminal portion of the serum concentration-time curve using linear regression with at least four last concentration-time points. The terminal-phase half-life $\left(t_{1 / 2}\right)$ was calculated as $0.693 / \lambda z$. The area under the curve from time zero to the last observed time $\left(\mathrm{AUC}_{0-t}\right)$ was calculated by the linear trapezoidal rule for ascending data points. The total area under the curve $\left(\mathrm{AUC}_{0-\infty}\right)$ was calculated as $\mathrm{AUC}_{0-t}+\mathrm{Ct} / \lambda z$, where $\mathrm{Ct}$ is the last measurable concentration. The apparent volume of distribution associated with the terminal phase $(V z)$ was calculated as $V z=\mathrm{CL} / \lambda z$, and the apparent total body clearance (CL) was calculated as $\mathrm{CL}=$ dose/AUC.

\section{Results and Discussion}

3.1. HPLC-MS/MS Condition Optimization. HPLC-MS/MS operation parameters were carefully optimized for the determination of photocyanine. Photocyanine is a typical sulfonate compound, which is usually ionized in negative mode. We tuned the mass spectrometer in both positive and negative ionization modes with ESI for photocyanine and found that both the signal intensity and ratio of signal to noise obtained in negative ionization mode were much greater than those in positive ionization mode, which was consistent with previous study on another sulfonate compound [19]. In the precursor ion full-scan spectra, the most abundant ions were protonated molecules with $\mathrm{m} / z$ ratios of 526.0 and 655.1 for photocyanine and IS, respectively. Parameters such as desolvation temperature, ESI source temperature, capillary voltage, and flow rate of desolvation gas and cone gas were all optimized to obtain the highest intensity of protonated analyte molecules. The product ion scan spectra showed high abundance fragment ions at $\mathrm{m} / \mathrm{z}$ values of 146.0 and 591.8 for photocyanine and IS, respectively. Multiple reaction monitoring (MRM) using the precursor $\rightarrow$ product ion transition of $\mathrm{m} / z 526.0 \rightarrow \mathrm{m} / z 146.0$ and $\mathrm{m} / z 655.1 \rightarrow \mathrm{m} / \mathrm{z}$ 591.8 was used for the quantification of photocyanine and IS, respectively.

The efficiency of the chromatographic separation of photocyanine and IS was evaluated using tests with different chromatographic columns and mobile phases. Photocyanine is amphoteric and relatively hydrophobic. We found that photocyanine displayed a very strong retention on BDS or XDB C18 columns, and little retention on C8 or SCX columns, which resulted in broad peak or substantial carry over. These columns have been tested without success. It has been shown that good chromatographic profiles of photocyanine and IS are obtained using a Waters XBridge C18 column $(50 \mathrm{~mm}$ $\times 4.6 \mathrm{~mm}, 5 \mu \mathrm{m}$ ), with retention times of 2.33 and $2.59 \mathrm{~min}$, respectively. The total analysis time per sample is $7 \mathrm{~min}$, which is much shorter than that of $45 \mathrm{~min}$ in previous study [18]. We also optimized the column temperature by observing the chromatographic peak and resolution and found that XBridge C18 column displayed well column performance at $60^{\circ} \mathrm{C}$. Optimization of the mobile phase is important for improving peak shape and detection sensitivity and for shortening the run time. We tested methanol, acetonitrile, and a mixture of the two as the organic modifier of the mobile phase, and we found that the peaks were more symmetric when methanol was used. Moreover, the chromatographic behavior of photocyanine subjected to mobile phases of different $\mathrm{pH}$ values was investigated, and we observed that deionized water (adjusted to $\mathrm{pH} 10.0$ with aqueous ammonia) improved the peak shape and significantly increased the signal intensity of the analyte. In order to further optimize the chromatographic condition, the peak symmetry factor of photocyanine was calculated in different percentage of MPB at the elution period 1.3-4.0 min. Symmetry factor of a peak is calculated by dividing $W_{0.05}$ by two-fold $f$, where $W_{0.05}$ is the width of the peak at $5 \%$ height and $f$ is the distance from the peak maximum to the leading edge of the peak, the distance being measured at a point $5 \%$ of the peak height from the baseline. As shown in Table 1, the optimal value was 95\%. Finally, the optimized gradient elution with deionized water (adjusted to $\mathrm{pH} 10.0$ with aqueous ammonia) and methanol at a flow rate of $0.6 \mathrm{~mL} / \mathrm{min}$ was established in this study.

3.2. Sample Preparation Procedure. Sample preparation is important for the HPLC-MS/MS assay. Liquid-liquid extraction (LLE) and solid-phase extraction (SPE) are techniques often used in the preparation of biological samples due to their ability to improve assay sensitivity $[20,21]$. SPE columns, including Strata, Strata-X, and Strata C18-E from Phenomenex (Torrence, CA, USA), OASIS WAX Cartridge, and Sep-pak C18 from Waters (Milford, MA, USA), were used for sample preparation in this study. However, photocyanine exhibited no elution due to its strong adsorption to the SPE columns. We also carried out LLE with ethyl acetate, nbutyl alcohol, and mixtures of these organic solvents with $\mathrm{n}$ hexane; however, we obtained low recovery and reproducibility using this procedure. Because HPLC-MS/MS quantification is highly specific and sensitive, protein precipitation (PPT) from the sample preparations was tried in the present study. We found that PPT was not only simple and efficient but also applicable to pharmacokinetic studies in which only $100 \mu \mathrm{L}$ of serum was used to obtain bioanalytical results. In addition, we observed that the linearity of photocyanine concentration in human serum with DMF was significantly improved than that of without DMF, indicating that DMF is conductive to maximize the release of photocyanine in PPT by inhibiting the binding of the drug to serum proteins, but there is no related report so far about the mechanism of DMF in PPT or drug release. Finally, we added $200 \mu \mathrm{L}$ DMF to the sample, and the effect of DMF here is consistent to that in previous study [18]. 
TABLE 2: Precision and accuracy of photocyanine.

\begin{tabular}{|c|c|c|c|c|}
\hline \multirow{2}{*}{ Concentration $(\mathrm{ng} / \mathrm{mL})$} & \multicolumn{2}{|c|}{ Intrabatch $(n=6)$} & \multicolumn{2}{|c|}{ Interbatch $(n=3)$} \\
\hline & Accuracy (\%) & RSD (\%) & Accuracy (\%) & RSD (\%) \\
\hline 60 & 103.72 & 4.91 & 105.62 & 4.72 \\
\hline 1000 & 101.98 & 1.29 & 100.52 & 5.93 \\
\hline 1600 & 107.54 & 3.31 & 101.18 & 8.53 \\
\hline
\end{tabular}

TABLE 3: Extraction recovery and matrix effects of photocyanine.

\begin{tabular}{lcc}
\hline $\begin{array}{l}\text { Concentration } \\
(\mathrm{ng} / \mathrm{mL})\end{array}$ & $\begin{array}{c}\text { Extraction recovery } \\
(\%) \\
(n=5)\end{array}$ & $\begin{array}{c}\text { Matrix effect }(\%) \\
(n=6)\end{array}$ \\
\hline 60 & 37.13 & 61.51 \\
1000 & 43.53 & 71.24 \\
1600 & 31.64 & 77.03 \\
\hline
\end{tabular}

3.3. Specificity. Specificity was determined by comparing the chromatograms of six different batches of blank human serum with the corresponding spiked serum. No interference from endogenous substances was observed at the respective retention times of photocyanine and IS (data not shown).

3.4. Linearity and $L L O Q$. The linear calibration curves were determined from the peak-area ratios (peak-area analyte/peak-area IS) versus concentration in human serum using a weighting factor $\left(1 / x^{2}\right)$, varying linearly over the concentration range tested $(20-2000 \mathrm{ng} / \mathrm{mL})$. As shown in Figure 3, the typical equation for the calibration curve for photocyanine was $y=0.421 x+0.00439(r=0.9965)$. The slopes of the equations were consistent with the calibration curves prepared on three separate days. The LLOQ in this study was $20 \mathrm{ng} / \mathrm{mL}$ for photocyanine, in which the $\mathrm{S} / \mathrm{N}$ was $>10$, and the precision of repeat injections was $8.26 \%$. Our method displayed a little higher sensitivity than the previous method, in which the LLOQ was $30 \mathrm{ng} / \mathrm{mL}$ [18].

3.5. Accuracy and Precision. The accuracy and precision of the method were determined by analyzing QC samples at three concentrations in six replicates. The intrabatch accuracy ranged from $101.98 \%$ to $107.54 \%$ at three concentrations, with precisions between $1.29 \%$ and $4.91 \%$. The interbatch accuracy varied from $100.52 \%$ to $105.62 \%$, with precisions ranging from $4.72 \%$ to $8.53 \%$ (Table 2). Thus, the present method has satisfactory accuracy, precision, and reproducibility.

3.6. Extraction Recovery and Matrix Effect. The extraction recoveries from QC samples at low, intermediate, and high concentrations ranged from $31.64 \%$ to $43.53 \%$ at three tested concentrations. We extracted the analyte from serum using protein precipitation and DMF in the present study, providing a simple and rapid method for the bioanalysis of photocyanine. In terms of matrix effects, the MF ranged from $61.51 \%$ to $77.03 \%$ at the three concentrations tested (Table 3), indicating that the coeluting substance only slightly influenced the ionization of the analyte.

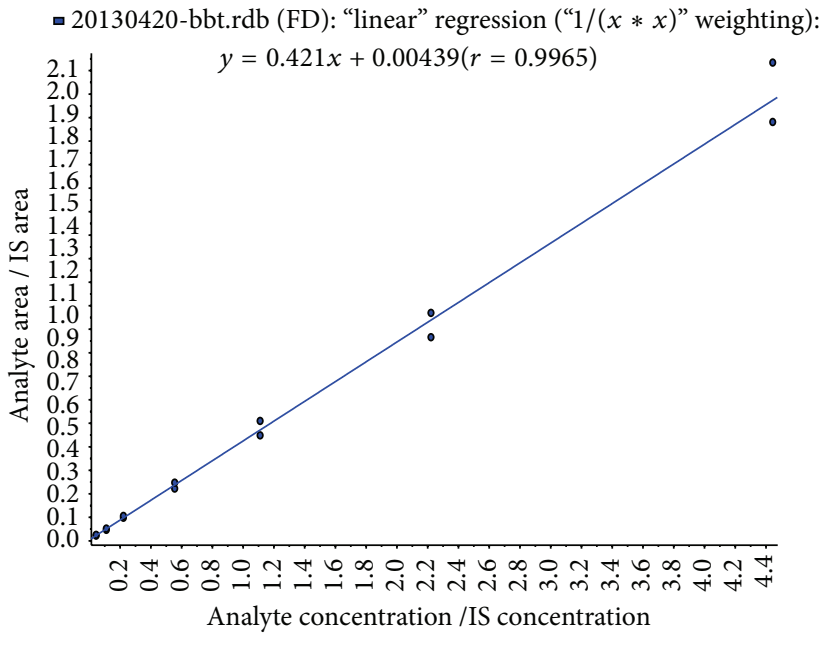

FIGURE 3: Linear calibration curve of photocyanine from the peakarea analyte/peak-area IS ratios versus concentrations in human serum using a weighting factor $\left(1 / x^{2}\right)$, varying linearly over the concentration range of $20-2000 \mathrm{ng} / \mathrm{mL}$. The equation for the curve was $y=0.421 x+0.00439(r=0.9965)$.

3.7. Stability. The results from the stability tests are presented in Table 4, and the data demonstrate a good stability of photocyanine throughout the steps of the determination. The method is therefore applicable to routine analysis.

3.8. Analysis of Patient Samples. The validated HPLC-MS/MS method described here was successfully applied to a pharmacokinetic study in 6 cancer patients following i.v. administration of $0.1 \mathrm{mg} / \mathrm{kg}$ photocyanine. A mean plasma concentration-time curve of a single dose of photocyanine is shown in Figure 4. This result revealed that our method was sufficiently sensitive to determine the photocyanine concentration in the serum of patients. The parameters of the pharmacokinetic analysis are shown in Table 5. The time of maximum plasma concentration $\left(T_{\max }\right)$ was $1.83 \pm 0.41 \mathrm{~h}$, the maximum plasma concentration $\left(C_{\max }\right)$ was $2465.3 \pm$ $723.0 \mathrm{ng} / \mathrm{mL}$, the half-life of drug elimination at the terminal phase $\left(t_{1 / 2}\right)$ was $74.62 \pm 13.29 \mathrm{~h}$, the area under the plasma concentration-time curve from $0 \mathrm{~h}$ to the time of the last detectable concentration $\left(\mathrm{AUC}_{0-t}\right)$ was $53137.2 \pm$ $20210.6 \mathrm{ng} \cdot \mathrm{mL}^{-1} \cdot \mathrm{h}$, the area under the plasma concentrationtime curve from $0 \mathrm{~h}$ to infinity $\left(\mathrm{AUC}_{0-\infty}\right)$ was $62634.6 \pm$ $25398.6 \mathrm{ng} \cdot \mathrm{mL}^{-1} \cdot \mathrm{h}$, the volume of distribution $\left(V_{d}\right)$ of photocyanine was $11.29 \pm 4.12 \mathrm{~L}$, the total clearance $(\mathrm{CL})$ was $0.11 \pm 0.04 \mathrm{~L} / \mathrm{h}$, and the mean residence time $(\mathrm{MRT})$ was $40.16 \pm 4.32 \mathrm{~h}$. 
TABLE 4: Stability of photocyanine under different storage conditions.

\begin{tabular}{|c|c|c|c|}
\hline Storage conditions & Concentration (ng/mL) & Accuracy $(\%)(n=6)$ & RSD (\%) \\
\hline \multirow{3}{*}{ Freeze-thaw three cycles } & 60 & 107.08 & 6.09 \\
\hline & 1000 & 101.20 & 4.14 \\
\hline & 1600 & 93.92 & 1.76 \\
\hline \multirow{3}{*}{$-80^{\circ} \mathrm{C}$ for 30 days } & 60 & 95.14 & 10.01 \\
\hline & 1000 & 89.56 & 1.85 \\
\hline & 1600 & 85.31 & 3.26 \\
\hline \multirow{3}{*}{ Post-preparative (room temperature for $24 \mathrm{~h}$ ) } & 60 & 102.52 & 12.12 \\
\hline & 1000 & 96.16 & 3.55 \\
\hline & 1600 & 102.15 & 2.00 \\
\hline
\end{tabular}

TABLE 5: Noncompartmental pharmacokinetic parameters of photocyanine in cancer patients after a single i.v. dose of $0.1 \mathrm{mg} / \mathrm{kg}$ photocyanine $(n=6)$.

\begin{tabular}{lc}
\hline Parameters & Mean $\pm \mathrm{SD}$ \\
\hline$T_{\max }(\mathrm{h})^{\mathrm{a}}$ & $1.83 \pm 0.41$ \\
$C_{\max }(\mathrm{ng} / \mathrm{mL})^{\mathrm{b}}$ & $2465.3 \pm 723.0$ \\
$t_{1 / 2}(\mathrm{~h})^{\mathrm{c}}$ & $74.62 \pm 13.29$ \\
$\mathrm{AUC}_{0-t}{ }^{\mathrm{d}}\left(\mathrm{ng} \cdot \mathrm{mL}^{-1} \cdot \mathrm{h}\right)$ & $53137.2 \pm 20210.6$ \\
$\mathrm{AUC}_{0-\infty}{ }^{\mathrm{e}}\left(\mathrm{ng} \cdot \mathrm{mL}^{-1} \cdot \mathrm{h}\right)$ & $62634.6 \pm 25398.6$ \\
$V_{d}(\mathrm{~L})^{\mathrm{f}}$ & $11.29 \pm 4.12$ \\
$\mathrm{CL}(\mathrm{L} / \mathrm{h})^{\mathrm{g}}$ & $0.11 \pm 0.04$ \\
$\mathrm{MRT}^{\mathrm{h}}(\mathrm{h})^{\mathrm{h}}$ & $40.16 \pm 4.32$ \\
\hline
\end{tabular}

${ }^{\mathrm{a}} T_{\max }$ : time to maximum concentration; ${ }^{\mathrm{b}} \mathrm{C}_{\max }$ : maximum plasma concentration; ${ }^{c} t_{1 / 2}$ : half-life of elimination; ${ }^{\mathrm{d}} \mathrm{AUC}_{0-t}$ : area under the concentration-time curve from time zero to the last quantifiable measurement; ${ }^{\mathrm{e}} \mathrm{AUC}_{0-\infty}$ : area under the concentration-time curve extrapolated to infinity; ${ }^{\mathrm{f}} V_{d}$ : volume of distribution; ${ }^{\mathrm{g}} \mathrm{CL}$ : total clearance; ${ }^{\mathrm{h}} \mathrm{MRT}$ : mean residence time.

\section{Conclusion}

A selective, sensitive, and rapid HPLC-MS/MS method for measuring photocyanine in human serum is described. In comparing this method with the analytical methods reported previously $[14,18]$, the present method proved superior with respect to higher simplicity of sample preparation, higher selectivity and sensitivity, and shorter chromatographic analysis time. The present description is the first to utilize the HPLC-MS/MS method for the pharmacokinetic study of photocyanine given by injection to cancer patients. $100 \mu \mathrm{L}$ human serum is sufficient for obtaining results in our pharmacokinetic study, indicating that the present method is applicable to human phase I clinical trials.

\section{Conflict of Interests}

The authors declare that there is no conflict of interests regarding the publication of this paper.

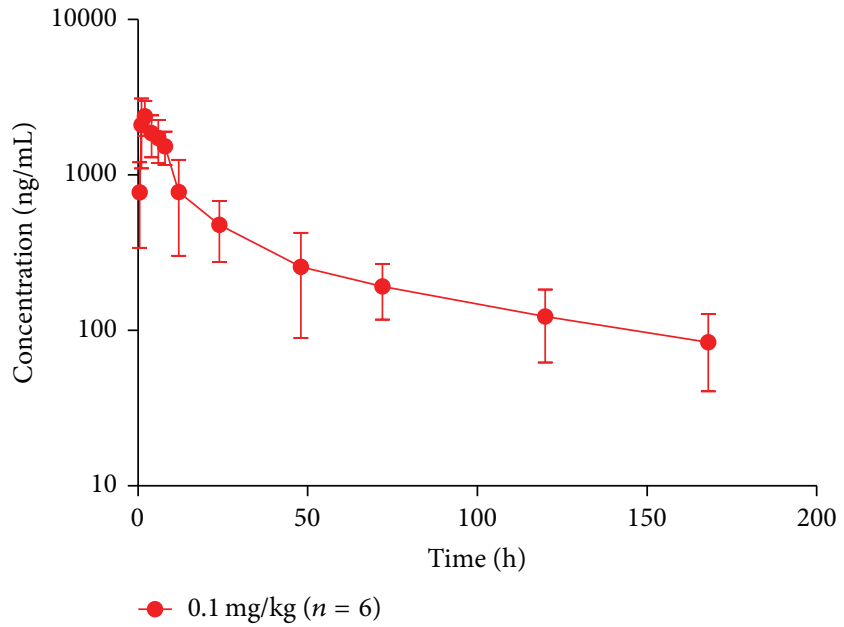

FIGURE 4: Mean serum concentration-time curve of photocyanine after $0.1 \mathrm{mg} / \mathrm{kg}$ single i.v. administration to cancer patients $(n=6)$.

\section{Authors' Contribution}

Bing-Tian Bi and Ben-Yan Zou contributed equally to this work.

\section{Acknowledgments}

Fujian Longhua Pharmaceutical Co. provided funding for the photocyanine analysis. There were no other sources of funding supporting this study.

\section{References}

[1] D. E. J. G. J. Dolmans, D. Fukumura, and R. K. Jain, "Photodynamic therapy for cancer," Nature Reviews Cancer, vol. 3, no. 5, pp. 380-387, 2003.

[2] B. C. Wilson, "Photodynamic therapy for cancer: principles," Canadian Journal of Gastroenterology, vol. 16, no. 6, pp. 393396, 2002.

[3] M. B. Vrouenraets, G. W. Visser, G. B. Snow, and G. A. van Dongen, "Basic principles, applications in oncology and improved selectivity of photodynamic therapy," Anticancer Research, vol. 23, no. 1B, pp. 505-522, 2003. 
[4] E. A. Lukyanets, "Phthalocyanines as photosensitizers in the photodynamic therapy of cancer," Journal of Porphyrins and Phthalocyanines, vol. 3, no. 6-7, pp. 424-432, 1999.

[5] N. Shirasu, S. O. Nam, and M. Kuroki, “Tumor-targeted photodynamic therapy," Anticancer Research, vol. 7, pp. 2823-2831, 2013.

[6] P. Mroz, A. Pawlak, M. Satti et al., "Functionalized fullerenes mediate photodynamic killing of cancer cells: type I versus type II photochemical mechanism," Free Radical Biology and Medicine, vol. 43, no. 5, pp. 711-719, 2007.

[7] R. Saini and C. Poh, "Photodynamic therapy: a review and its prospective role in the management of oral potentially malignant disorders," Oral Diseases, vol. 5, pp. 440-451, 2013.

[8] A. E. O’Connor, W. M. Gallagher, and A. T. Byrne, "Porphyrin and nonporphyrin photosensitizers in oncology: preclinical and clinical advances in photodynamic therapy," Photochemistry and Photobiology, vol. 85, no. 5, pp. 1053-1074, 2009.

[9] H. Yaku, T. Fujimoto, T. Murashima, D. Miyoshi, and N. Sugimoto, "Phthalocyanines: a new class of G-quadruplex-ligands with many potential applications," Chemical Communications, vol. 48, no. 50, pp. 6203-6216, 2012.

[10] I. Yoon, J. Z. Li, and Y. K. Shim, "Advance in photosensitizers and light delivery for photodynamic therapy," Clinical Endoscopy, vol. 46, pp. 7-23, 2013.

[11] I. N. Skidan, A. I. Bobruskin, A. E. Guliaev et al., "Optimization of "Photosens" pharmakokinetics by biodegradable nanospheres," Antibiotiki i khimioterapiia, vol. 46, no. 4, pp. 610, 2001.

[12] E. D. Baron, C. L. Malbasa, D. Santo-Domingo et al., "Silicon phthalocyanine (pc 4) photodynamic therapy is a safe modality for cutaneous neoplasms: results of a phase 1 clinical trial," Lasers in Surgery and Medicine, vol. 42, no. 10, pp. 728-735, 2010.

[13] J. D. Miller, E. D. Baron, H. Scull et al., "Photodynamic therapy with the phthalocyanine photosensitizer Pc 4: the case experience with preclinical mechanistic and early clinicaltranslational studies," Toxicology and Applied Pharmacology, vol. 224, no. 3, pp. 290-299, 2007.

[14] W. G. Love, S. Duk, R. Biolo, G. Jori, and P. W. Taylor, "Liposome-mediated delivery of photosensitizers: localization of zinc (II)-phthalocyanine within implanted tumors after intravenous administration," Photochemistry and Photobiology, vol. 63, no. 5, pp. 656-661, 1996.

[15] J. Huang, N. Chen, E. Liu, J. Xue, and S. Yang, "Preparation of phthalocyanine zinc and its application as anticancer drug," Chinese Patent, Patent No. ZL96117137.5. State Intellectual Property Office of the People's Republic of China.

[16] J. Huang, N. Chen, E. Liu, J. Xue, and S. Yang, "Synthesis, peculiarity and anti-tumor potency of the double affinity photosensitizer-zinc phthalocyanine," Science in China Series B: Chemistry, vol. 30, pp. 481-488, 2000.

[17] J. Huang, N. Chen, E. Liu et al., "Metal phthalocyanine as photosensitizer for photodynamic therapy (PDT)-preparation, characterization and anticancer activities of an amphiphilic phthalocyanine ZnPcS2P2," Science in China, Series B: Chemistry, vol. 44, no. 2, pp. 113-122, 2001.

[18] S. Li, F. Jian, J. Zhan, B. Zou, and H. Liao, "Determination of photocyanine in human serum by HPLC and application to pharmacokinetic study," Journal of Chromatographic Science, pp. 1-7, 2013.

[19] X. Hu, Y. Zheng, G. Wu et al., "Optimization of solid phase extraction clean up and validation of quantitative determination of carbazochrome sodium human plasma by liquid chromatography-electrospray mass spectrometry," Journal of Chromatography B, vol. 951-952, pp. 1-6, 2014.

[20] V. Paiano, E. Fattore, A. Carrà, C. Generoso, R. Fanelli, and R. Bagnati, "Liquid chromatography-tandem mass spectrometry analysis of perfluorooctane sulfonate and perfluorooctanoic acid in fish fillet samples," Journal of Analytical Methods in Chemistry, vol. 1, no. 1, Article ID 719010, 2012.

[21] Z. Xie, Q. Liu, Z. Liang et al., "The GC/MS analysis of volatile components extracted by different methods from Exocarpium Citri Grandis," Journal of Analytical Methods in Chemistry, vol. 2013, Article ID 918406, 8 pages, 2013. 

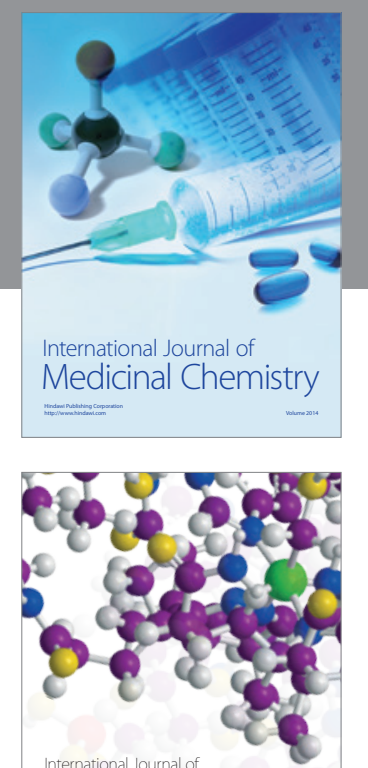

\section{Carbohydrate} Chemistry

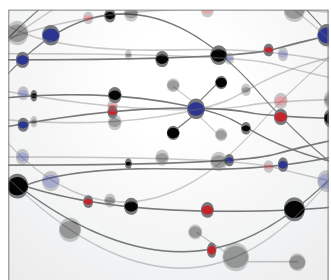

The Scientific World Journal
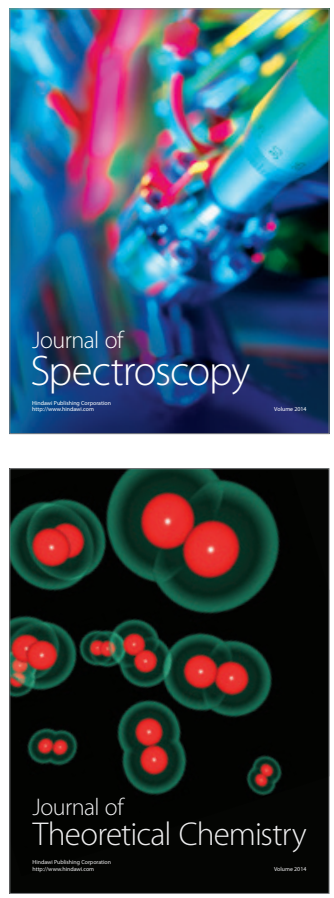
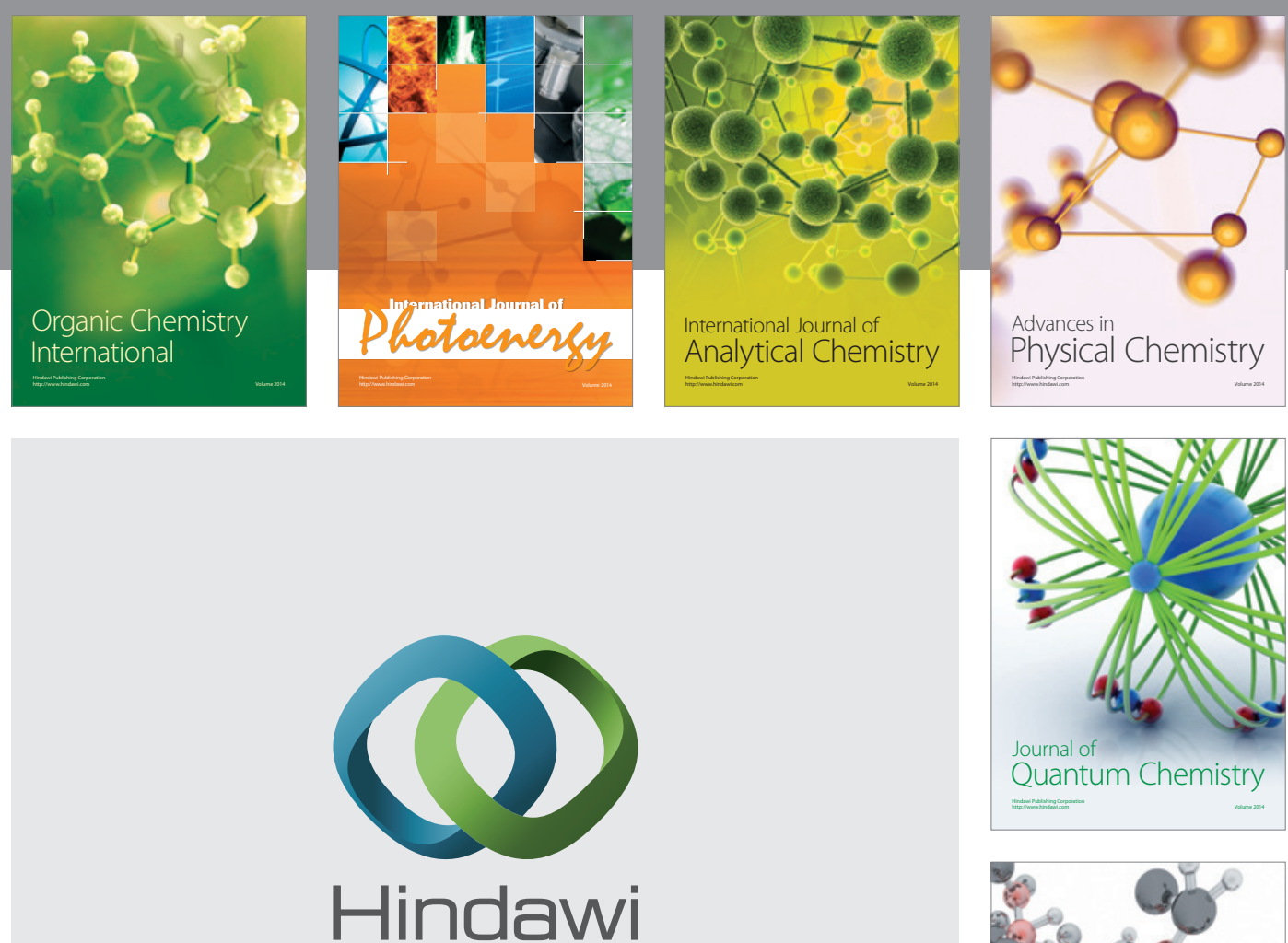

Submit your manuscripts at

http://www.hindawi.com

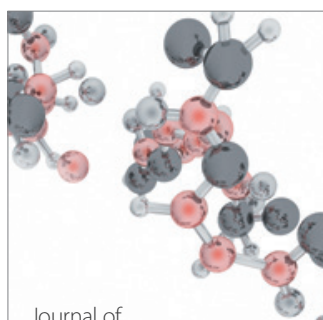

Analytical Methods

in Chemistry

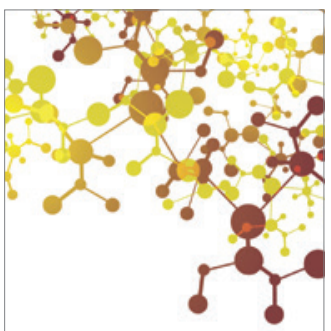

Journal of

Applied Chemistry

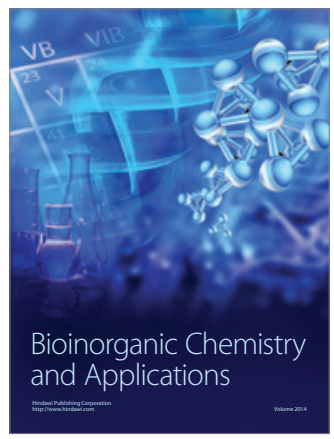

Inorganic Chemistry
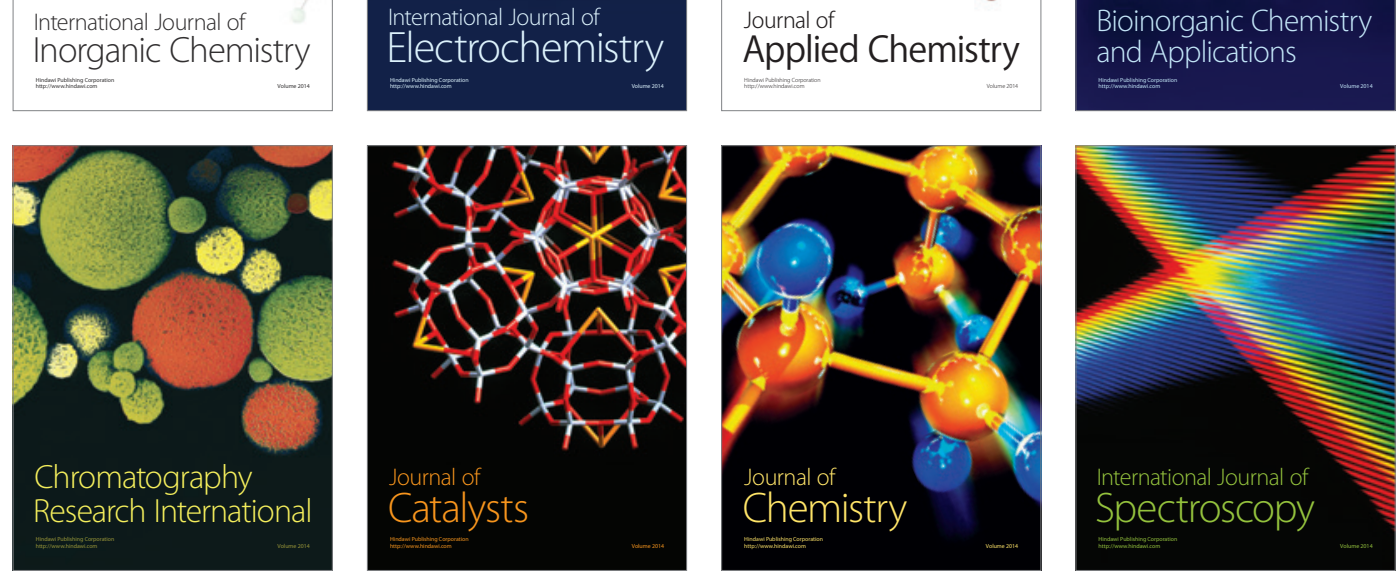